\title{
Large-scale association study on daily weight gain in pigs reveals overlap of genetic factors for growth in humans
}

\author{
Zexi Cai ${ }^{*}$, Ole Fredslund Christensen ${ }^{1}$, Mogens Sand $\varnothing ~ L u n d{ }^{1}$, Tage Ostersen² and Goutam Sahana
}

\begin{abstract}
Background: Imputation from genotyping array to whole-genome sequence variants using resequencing of representative reference populations enhances our ability to map genetic factors affecting complex phenotypes in livestock species. The accumulation of knowledge about gene function in human and laboratory animals can provide substantial advantage for genomic research in livestock species.

Results: In this study, 201,388 pigs from three commercial Danish breeds genotyped with low to medium (8.5k to 70k) SNP arrays were imputed to whole genome sequence variants using a two-step approach. Both imputation steps achieved high accuracies, and in total this yielded 26,447,434 markers on 18 autosomes. The average estimated imputation accuracy of markers with minor allele frequency $\geq 0.05$ was 0.94 . To overcome the memory consumption of running genome-wide association study (GWAS) for each breed, we performed within-breed subpopulation GWAS then within-breed meta-analysis for average daily weight gain (ADG), followed by a multi-breed meta-analysis of GWAS summary statistics. We identified 15 quantitative trait loci (QTL). Our post-GWAS analysis strategy to prioritize of candidate genes including information like gene ontology, mammalian phenotype database, differential expression gene analysis of high and low feed efficiency pig and human GWAS catalog for height, obesity, and body mass index, we proposed MRAP2, LEPROT, PMAIP1, ENSSSCG00000036234, BMP2, ELFN1, LIG4 and FAM155A as the candidate genes with biological support for ADG in pigs.

Conclusion: Our post-GWAS analysis strategy helped to identify candidate genes not just by distance to the lead SNP but also by multiple sources of biological evidence. Besides, the identified QTL overlap with genes which are known for their association with human growth-related traits. The GWAS with this large data set showed the power to map the genetic factors associated with ADG in pigs and have added to our understanding of the genetics of growth across mammalian species.
\end{abstract}

Keywords: GWAS, Large-scale association study, Pig breeding, Imputation, Candidate genes

\section{Background}

The number of genome-wide association studies (GWAS) has grown rapidly over the last decade to establish a link between genetic variants and complex traits in humans

\footnotetext{
*Correspondence: zexi.cai@ggg.au.dk

${ }^{1}$ Center for Quantitative Genetics and Genomics, Aarhus University, 8830 Tjele, Denmark

Full list of author information is available at the end of the article
}

and agricultural species. Genotype data for GWAS are usually generated using cost-effective genotyping arrays of common single nucleotide polymorphism (SNP) variants. The use of imputed whole-genome sequencing (WGS) is routine in human GWAS [1] due to the availability of whole-genome haplotype reference panels. A similar reference panel for cattle is available and routinely used in cattle GWAS studies [2, 3]. However, the imputation of a SNP array to WGS for GWAS in pigs is 
still not common. One reason could be the absence of an international haplotype panel in pigs. Imputation from a low-density marker set to a high-density marker set, and even up to WGS level, has shown high accuracy at an affordable cost for large-scale GWAS [3-6] and investigation of the genetic architecture of complex traits [7-9]. Normally, the reference panel for imputation requires a large number of individuals. However, the whole genome sequencing of many animals is still economically prohibitive. A previous study [10] showed the advantage of a two-step imputation strategy in cattle, where step 1: impute a low-density (50k) SNP array marker set to a high-density (700k) SNP array marker set; and step 2: impute the imputed high-density marker set to WGS. Brøndum et al. [11] showed that a multi-breed reference panel can increase imputation accuracy in cattle. Both strategies, namely the two-step imputation and multibreed reference population, can also be used to increase imputation accuracy in other livestock species like pigs. Unlike pure breeding in dairy cattle, two-way and three-way crosses are routinely used to produce slaughter pigs. Although we know that multi-breed reference could improve imputation accuracy, it is worth examining whether available high-density (HD) genotypes from crossbred pigs can be used as the intermediate reference panel for purebred pigs.

The growth rate is an important trait in pig breeding, as it is directly linked to economic returns. Average daily gain (ADG) is one of the most important indicators of the growth rate and indicates the time required for pigs to achieve the targeted market weight [12]. Previous studies have shown the complex genetic architecture of ADG [13], which puts a limit on how precisely the quantitative trait loci (QTL) can be determined. Approximately 753 QTL for ADG spread across all chromosomes in pigs are reported in the QTL database (queried in July 2020) [14]. Recently, Falker-Gieske et al. performed GAWS using imputed WGS to identify QTLs on chromosome 2, 4 and 7 for ADG [15]. Similarly, a larger number of genetic factors affecting growth-related traits in humans and other mammalian species are known [3, 16, 17]. Therefore, precise mapping of genetic factors for ADG in pigs could highlight the common genetic factors affecting growth in humans and other mammalian species. Furthermore, in pig breeding, mapped WGS variants, if included in a genomic selection marker panel, may increase the prediction accuracy for ADG [18].

The knowledge gained from non-human species could bring new insights for human studies. Mice are one common model species for human research $[19,20]$, but the scale of species could expand to other rodent species [21]. Researches have used model species to study specific human diseases, e.g., ferrets, as a model for human respiratory disease [22], and sheep as a model for human asthma and other respiratory diseases [23]. Similarly, for quantitative traits, meta-analysis of cattle stature revealed the genetic similarity between human height and cattle stature [3]. A pig model is used to study several human traits [24-27]. Therefore, the mapping of genetic factors for ADG in pigs could be utilized to add to our knowledge about growth traits in humans.

The aim of this study was to detect the WGS variants associated with ADG in pigs, and to study whether the candidate genes underlying these associated variants in pigs for ADG are known for their association with growth-related traits in humans. To achieve this goal, we used phenotypes and SNP array genotypes from 201,388 animals from three Danish pig breeds. We divided each breed into subpopulations to run GWAS and then carried out a within-breed meta-analysis, followed by a multi-breed meta-analysis. In the post-GWAS analyses, we examined whether the identified candidate genes in pigs are known to be related to growth-related phenotypes in humans.

\section{Results \\ Imputation to WGS level}

After imputation of SNP array genotyped animals to WGS variants, we obtained 26,447,434 markers on 18 autosomes. In this study, we used HD genotyped crossbred pigs as an intermediate reference. The estimated imputation accuracy $\left(R^{2}\right.$ reported by Minimac 4$)$ is comparable to the cattle study by Daetwyler et al. [2]. The average estimated imputation accuracy for the markers with minor allele frequency $(\mathrm{MAF})>=0.05$ reached 0.94 . If the markers with estimated imputation accuracy below 0.4 are filtered out, the average estimated imputation accuracy reaches above 0.9 for all MAF classes (Fig. 1). Moreover, as shown in Fig. 1, most of the inaccurate imputed markers $\left(R^{2}<=0.4\right)$ are markers with low MAF $(0-0.05)$, which are very challenging to impute accurately due to the lack of haplotype in the reference population. After quality filtering with MAF $(0.5 \%)$ and HardyWeinberg proportions $\left(p<10^{-6}\right)$, the WGS marker set for association study was 12,596,412 for Duroc, 18,654,181 for Landrace, and 14,522,325 for Yorkshire breeds. As the current computational facility available to us was limited, we could not run GWAS analysis including all animals from a breed, and we therefore adopted the strategy to split each of the breeds into three subpopulations, and then combined results using within-breed meta-analysis.

\section{Association analysis for average daily gain in Duroc}

In Duroc, we identified one QTL on chromosome 1 in the within-breed meta-analysis $(53,054,787$ 54,008,032; Fig. 2, Supplementary Figs. S1 and S4 and 


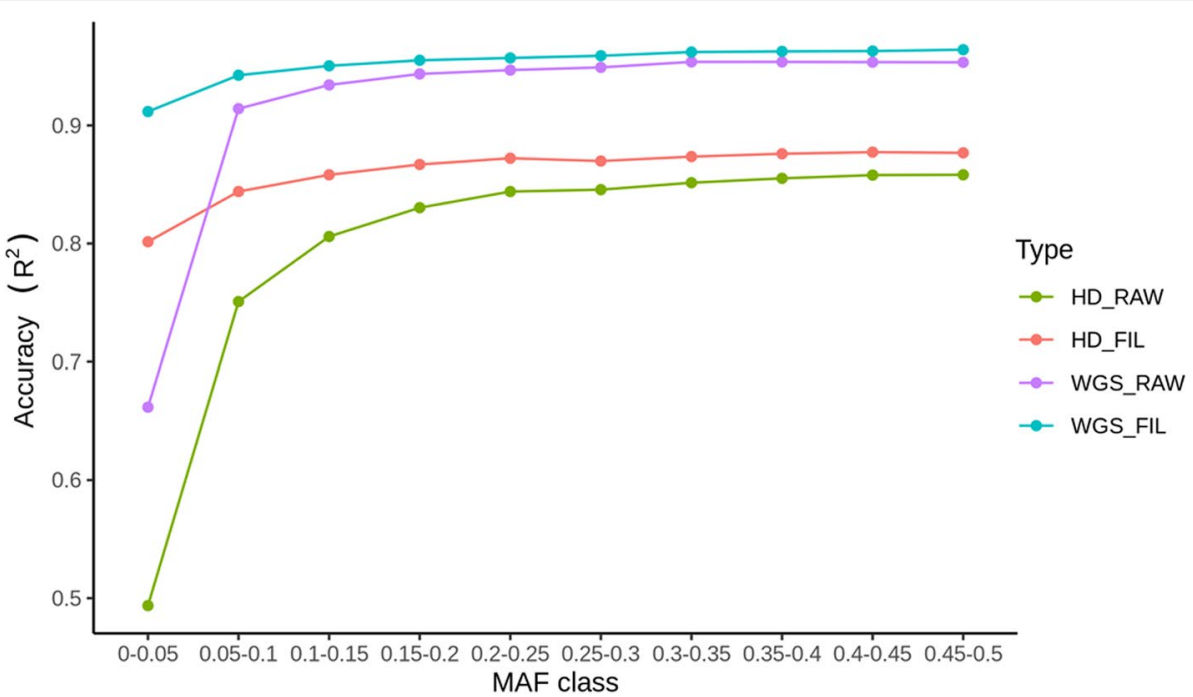

Fig. 1 Average estimated imputation accuracy across different minor allele frequencies (MAF); HD_RAW is for imputation from low-density chip to HD (all markers); HD_FIL is for imputation from $60 \mathrm{k}$ to HD for markers with estimated imputation accuracy $\geq 0.4$; WGS_RAW is for imputation from imputed HD to WGS (all markers); and WGS_FIL is for imputation from imputed HD to WGS for markers with estimated imputation accuracy $\geq 0.4$

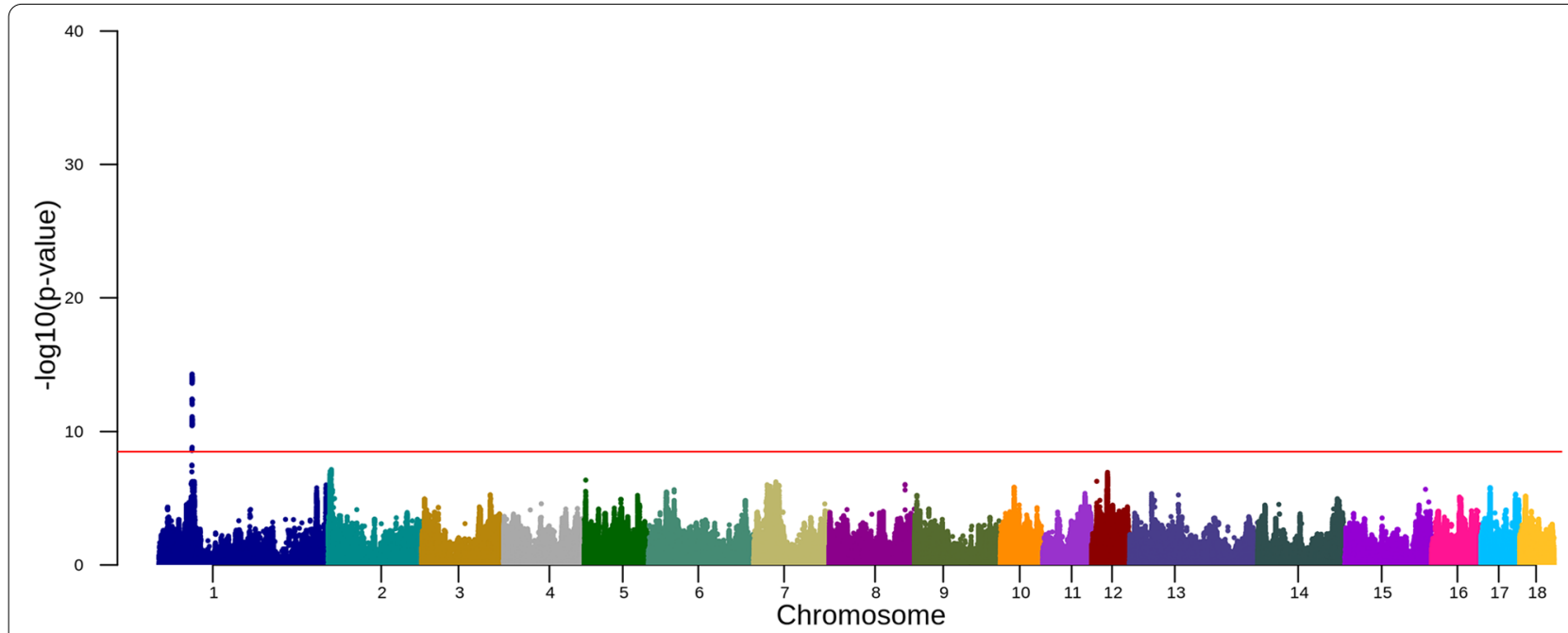

Fig. 2 Manhattan plot for the association of SNPs with daily weight gain in Duroc. The red horizontal line indicates a genome-wide significance level $[-\log 10(p$-value $)=8.5]$

Supplementary Table S1). Splitting into subsets of data based on birth years, we did not identify any QTL in animals born in 2015-2016 and 2017-2018 (Fig. S1a and $\mathrm{b})$. We only detected an association signal in animals born before 2015 (Fig. S1c), and this is the same as in the within-breed meta-analysis (Fig. 2). The lead SNP of this association is 1: 53289914 (rs344908085) with $-\log _{10}(p$-value $)=14.30$. This SNP is located in the intron of the MRAP2 gene, which encodes Melanocortin-2 receptor accessory protein 2 .

\section{Association analysis of average daily gain in Landrace}

In Landrace, we located five QTL on five chromosomes in the within breed meta-analysis (Fig. 3, Table 1, Supplementary Figs. S2 and S5 and Supplementary Table S1). The strongest association signal was 1: 160174493 $\left(\right.$ rs343467711, $-\log _{10}(p$-value $\left.)=29.19\right)$ with the nearest gene being $\mathrm{CDH} 2 \mathrm{O}$. The second strongest association signal was on chromosome 12, where the lead SNP was 12: 3639288 ( $r$ 1109299516) within the intron of TK1. The third-strongest association signal was 6: 146958866 


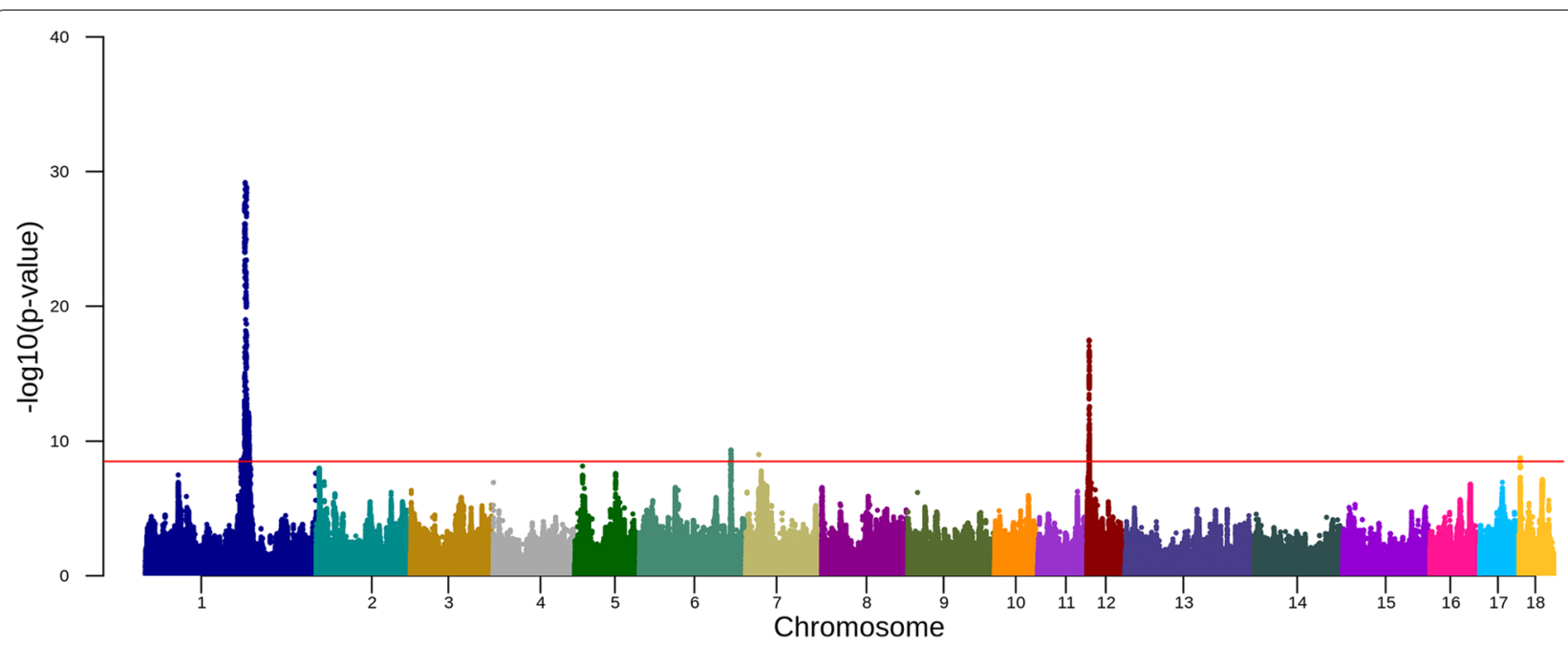

Fig. 3 Manhattan plot for the association of SNPs with daily weight gain in Landrace. The red horizontal line indicates a genome-wide significance level $[-\log 10(p$-value $)=8.5]$

Table 1 Genomic regions identified by within-breed meta-analysis from subpopulation genome-wide association analysis of daily weight gain in Landrace

\begin{tabular}{lllllll}
\hline Chr & Lead SNP location (bp) & Region & rs id of lead SNP & ${\text { - } \log _{10} \text { ( } \boldsymbol{p} \text {-value) }}$ & Annotation of lead SNP & Nearest gene \\
\hline 1 & $160,174,493$ & $159,655,745 \sim 160,704,722$ & rs343467711 & 29.19 & intergenic_variant & CDH20 \\
6 & $146,958,866$ & $146,482,476 \sim 147,783,245$ & rs334716220 & 9.32 & intergenic_variant & LEPROT \\
7 & $21,015,982$ & $20,500,737 \sim 21,587,268$ & rs697892846 & 9.01 & intergenic_variant & ABT1 \\
12 & $3,639,288$ & $3,530,556 \sim 4,277,570$ & rs1 109299516 & 17.48 & intron_variant & TK1 \\
18 & $2,162,425$ & $1,301,989 \sim 2,856,015$ & rs793031877 & 8.74 & intergenic_variant & RNF32 \\
\hline
\end{tabular}

(rs334716220), which was annotated as an intergenic variant with LEPROT as the closest gene. On chromosome 7, the lead SNP was 7: 21015982 (rs697892846) near to $A B T 1$. On chromosome 18, the lead SNP was 18:2162425. This lead SNP is an intergenic variant with RNF32 as the nearest gene.

\section{Association analysis of average daily gain in Yorkshire}

In Yorkshire, we identified nine QTL on eight different chromosomes in the within breed meta-analysis (Fig. 4, Table 2, Supplementary Figs. S3 and S6 and Supplementary Table S1). The strongest signal was 1:160950166 with $-\log _{10}(p$-value) equal to 33.78 . This lead SNP is an intergenic variant and the nearest gene is ENSSSCG00000036234. The QTL interval in Yorkshire largely overlapped with the QTL interval on chromosome 1 of Landrace, however the nearest gene in these two breeds are different. The second-strongest association signal was 12:15311500 located in the intron of TACO1. The other lead SNP on chromosome 12 was 12:43812683 with NF1 as the nearest gene. The fourth-strongest association signal was on chromosome 17 with 17:15758097 (rs694525579) as the lead SNP and located at the intron of $B M P 2$. The lead SNP on chromosome 11 was located at 75,538,956 bp (rs319374568) which is within the coding sequence of LIG4. On chromosomes 3, 4, 7 and 13, we also found association signals with ELFN1, RB1CC1, ENSSSCG00000031184, and ENSSSCG00000037247 as the nearest genes, respectively (Table 2).

\section{Multi-breed meta-analysis of average daily gain of three breeds}

The total number of QTL detected in multi-breed metaanalysis of three breeds contains 7 QTL on chromosomes 1, 4, 11 and 12 (Fig. 5, Table 3 and Supplementary Table S1). The multi-breed meta-analysis of three breeds did not reveal new QTL compared to the within-breed meta-analysis (Table 3). However, the lead SNP and the detailed QTL interval suggested by the multi-breed meta-analysis of three breeds is slightly different from the within-breed meta-analysis within each breed (Table 3). In the list of the nearest genes from multi-breed metaanalysis, we found some nearest genes are different from the within-breed meta-analysis. On chromosome 1, the 


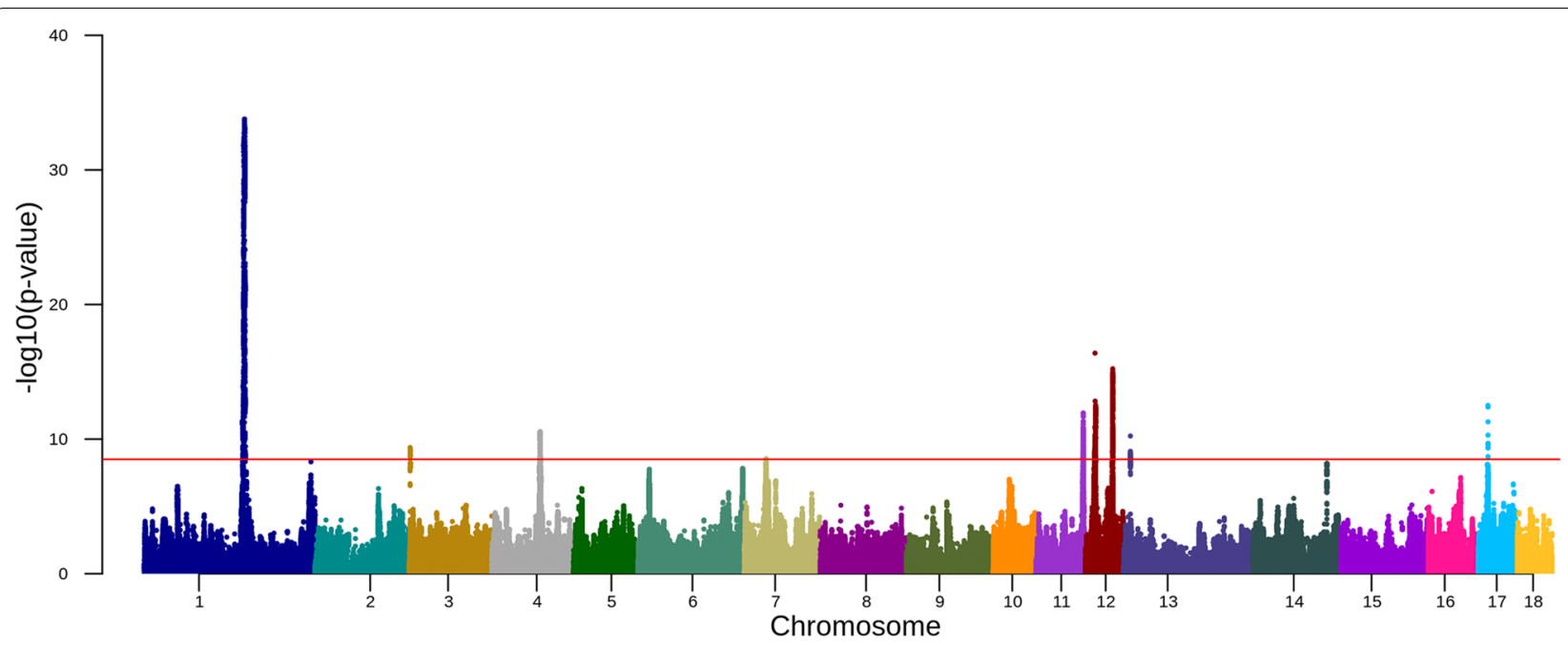

Fig. 4 Manhattan plot for the association of SNPs with daily weight gain in Yorkshire. The red horizontal line indicates a genome-wide significance level $[-\log 10(p$-value $)=8.5]$

Table 2 Genomic regions identified by within-breed meta-analysis from subpopulation genome-wide association analysis of daily weight gain in Yorkshire

\begin{tabular}{lllllll}
\hline Chr & $\begin{array}{l}\text { Location of } \\
\text { lead SNP (bp) }\end{array}$ & Region & rs id of lead SNP & ${\boldsymbol{- ~} \log _{10}(\boldsymbol{p} \text {-value) }}$ & Annotation of the lead SNP & Nearest gene \\
\hline 1 & $160,950,166$ & $160,704,722 \sim 161,224,815$ & NA & 33.78 & intergenic_variant & ENSSSCG00000036234 \\
3 & $1,223,929$ & $1,068,917 \sim 2,206,109$ & rs343847926 & 9.38 & upstream_gene_variant & ELFN1 \\
4 & $77,498,483$ & $77,174,471 \sim 77,837,179$ & rs345856128 & 10.54 & intergenic_variant & RB1CC1 \\
7 & $34,791,135$ & $34,405,000 \sim 35,135,343$ & rs319751202 & 8.53 & intron_variant & ENSSSCG00000031184 \\
11 & $75,538,956$ & $75,215,348 \sim 76,531,480$ & rs319374568 & 11.94 & synonymous_variant & LIG4 \\
12 & $15,311,500$ & $15,084,562 \sim 15,735,657$ & NA & 16.39 & intron_variant & TACO1 \\
12 & $43,812,683$ & $43,485,392 \sim 44,078,471$ & NA & 15.23 & intergenic_variant & NF1 \\
13 & $10,845,327$ & $10,754,226 \sim 11,415,685$ & NA & 10.23 & intergenic_variant & ENSSSCG00000037247 \\
17 & $15,758,097$ & $15,490,020 \sim 16,171,660$ & rs694525579 & 12.50 & intron_variant & BMP2 \\
\hline
\end{tabular}

multi-breed meta-analysis suggested the nearest gene detected in Yorkshire instead of the one detected in Landrace. On chromosome 11, the multi-breed meta-analysis suggested a new nearest gene FAM155A other than LIG4 suggested from the within-breed meta-analysis of Yorkshire. For the first QTL on chromosome 12, the multi-breed meta-analysis suggested a new nearest gene TNRC6C rather than TK1 suggested from the withinbreed meta-analysis of Landrace. And for the third QTL on chromosome 12, the multi-breed meta-analysis also suggested a new nearest gene WSB1 instead of NF1 suggested from the within-breed meta-analysis of Yorkshire.

\section{Post-GWAS analyses}

The candidate gene on chromosome 1 in Duroc and multi-breed meta-analysis of three breeds, $M R A P 2$, belongs to the gene ontology (GO) terms "energy reserve metabolic process" (GO: 0006112) and "feeding behavior" (GO: 0007631). In the Mammalian Phenotype database (MPD), multiple phenotype terms are related to obesity or body size for $M R A P 2$, e.g., "increased body weight", "obese", "increased total body fat amount", and "increased food intake". The candidate gene on chromosome 1 in Yorkshire and multi-breed meta-analysis, ENSSSCG00000036234, is glutamate decarboxylase 1-like. The GO term showed that this gene responds to the "carboxylic acid metabolic process" (GO: 0019752). $L E P R O T$, the candidate gene on chromosome 6 in Landrace, belongs to the GO term "negative regulation of growth hormone receptor signaling pathway" (GO: 0060400). From MPD entry, a mutation in LEPROT could cause "increased food intake" or "decreased total body fat amount". ELFN1, the nearest gene for ADG in Yorkshire showed a MPD entry as "increased lean body mass". LIG4, the nearest gene for the lead SNP on chromosome 11 of Yorkshire showed a MPD entry as "decreased body 


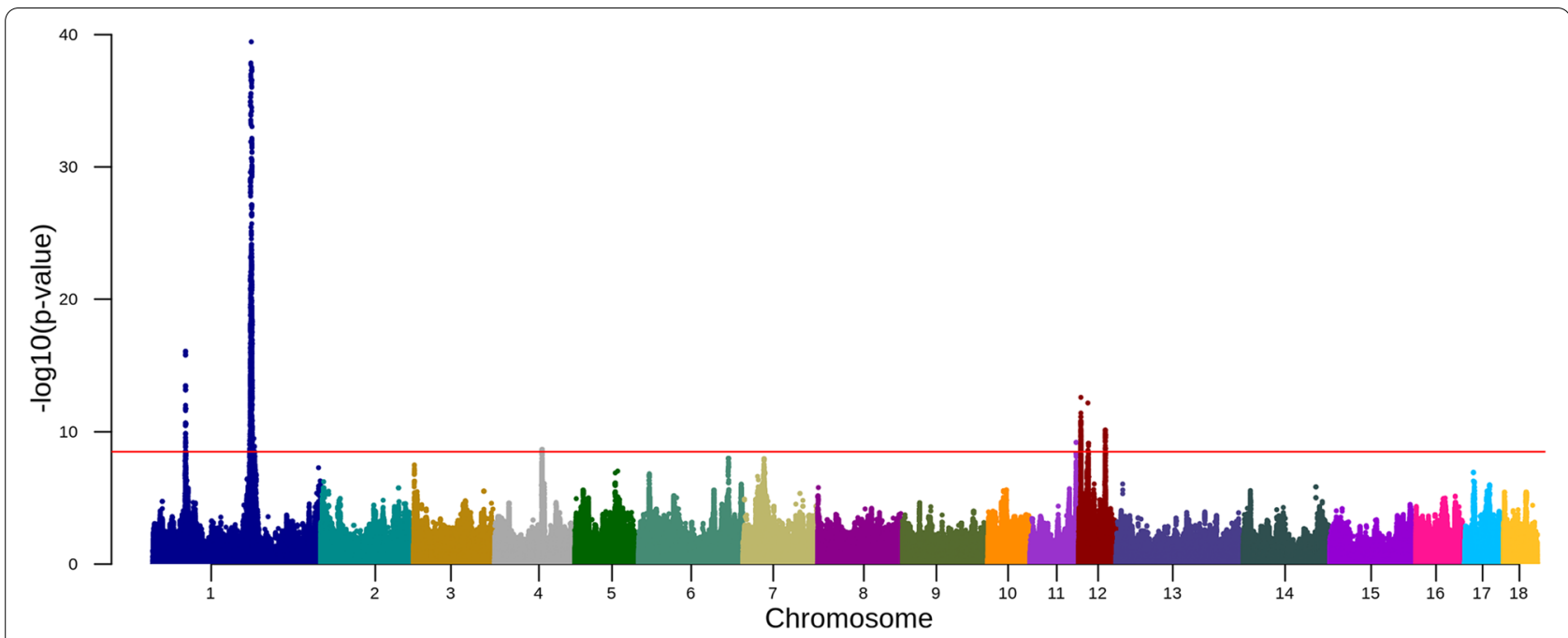

Fig. 5 Manhattan plot for the Meta-analysis of SNPs with daily weight gain of three breeds. The red horizontal line indicates a genome-wide significance level $\left[-\log _{10}(p\right.$-value $\left.)=8.5\right]$

Table 3 Genomic regions identified by multi-breed meta-analysis of daily weight gain in three breeds

\begin{tabular}{lllllll}
\hline Chr & Lead SNP location (bp) & Region & rs id of lead SNP & ${\text { - } \log _{\mathbf{1 0}}(\boldsymbol{p} \text {-value) }}$ & Annotation of lead SNP & Nearest gene \\
\hline 1 & $160,827,384$ & $160,137,530 \sim 161,334,328$ & rs334720929 & 39.46 & intergenic_variant & ENSSSCG00000036234 \\
1 & $53,289,914$ & $53,184,709 \sim 54,008,032$ & rs344908085 & 16.09 & intron_variant & MRAP2 \\
4 & $77,498,071$ & $77,031,250 \sim 77,782,587$ & rs345469413 & 8.66 & intergenic_variant & RB1CC1 \\
11 & $75,215,102$ & $74,903,365 \sim 76,080,211$ & rs338148206 & 9.20 & intron_variant & FAM155A \\
12 & $3,876,962$ & $3,604,107 \sim 4,277,570$ & NA & 12.61 & intron_variant & TNRC6C \\
12 & $15,311,500$ & $15,084,562 \sim 15,735,657$ & NA & 12.18 & intron_variant & TACO1 \\
12 & $43,848,701$ & $43,594,176 \sim 44,823,295$ & rs324835464 & 10.13 & intergenic_variant & WSB1 \\
\hline
\end{tabular}

size". And BMP2 on chromosome 17 of Yorkshire showed a MPD entry as "decreased body weight".

We could not established biological support for most of the nearest gene with GO, Kyoto Encyclopedia of Genes and Genomes (KEGG) [28], and MPD. Therefore, we downloaded three RNAseq data sets to find further possible candidate genes (Supplementary Table S2). Although the definition of the trait between GWAS and RNAseq dataset and also the different population architecture could largely reduce the power of this strategy, finding the overlap between GWAS result and RNAseq from different sources could take advantage of the public available dataset to provide biological insight into the GWAS signals. These three data sets included longissimus thoracis muscle tissue [29], liver tissue [30], and longissimus dorsi muscle tissue with liver tissue [31] classifying pigs based on their feed efficiency. We searched for differential expressed genes (DEG) in each QTL region to identify candidate genes with more support from functional activity rather than just picking the nearest gene of the lead SNP (Table 4). For Duroc, no gene within the QTL interval was expressed differently between low and high feed efficiency animals. In Landrace, H1-4 located within the QTL region on chromosome 7 and MNX1 located within the QTL region on chromosome 18 showed differential expression in the liver [31]. The QTL interval at chromosome 1 harbored one DEG between low and high feed efficiency animals, PMAIP1. This gene is located in the QTL interval in Yorkshire and multi-breed meta-analysis. In Yorkshire, six other genes in the QTL intervals were DEGs in the liver [31]. They are PMAIP1, RGS20, FAM155A, ENSSSCG00000017285, ENSSSCG00000049912 and NR1D2. The QTL interval from multi-breed metaanalysis of three breeds identified four more genes overlapping with the DEGs, ENSSSCG00000043998, $S L C 13 A 2, S E B O X$ and $V T N$. 
Table 4 The differentially expressed genes located within any of the QTL intervals for average daily weight gain in three pig breeds and multi-breed meta-analysis

\begin{tabular}{llll}
\hline Gene & log $_{\mathbf{2}}$ fold change & FDR $^{\mathbf{a}}$ & Breed \\
\hline PMAIP1 & -1.02 & $4.08 \mathrm{e}-7$ & Yorkshire and Meta-analysis \\
ENSSSCG00000043998 & -1.02 & $7.54 \mathrm{e}-8$ & Meta-analysis \\
RGS20 & -1.42 & $2.38 \mathrm{e}-2$ & Yorkshire \\
H1-4 & -1.37 & $6.76 \mathrm{e}-5$ & Landrace \\
FAM155A & -1.41 & $1.18 \mathrm{e}-2$ & Yorkshire and Meta-analysis \\
ENSSSCG00000017285 & -1.64 & $1.06 \mathrm{e}-3$ & Yorkshire and Meta-analysis \\
ENSSSCG00000049912 & -1.11 & $2.15 \mathrm{e}-3$ & Yorkshire and Meta-analysis \\
SLC13A2 & -1.26 & $1.98 \mathrm{e}-2$ & Meta-analysis \\
SEBOX & 1.32 & $7.92 \mathrm{e}-4$ & Meta-analysis \\
VTN & 1.19 & $1.96 \mathrm{e}-5$ & Meta-analysis \\
NR1D2 & -1.23 & $3.58 \mathrm{e}-16$ & Yorkshire \\
MNX1 & 1.39 & $8.74 \mathrm{e}-3$ & Landrace
\end{tabular}

a FDR false discovery rate

\section{Overlap with the associations reported for human growth-related traits}

Daily weight gain is a growth-related trait. Therefore, the candidate genes identified for ADG in pigs could overlap with genes in humans for BMI, height, and obesity. If we see homologous genes across mammalian species, that will increase the confidence that the identified candidate genes are true. We checked genes in the QTL interval with BMI (EFO_0004340), height (EFO_0004339), and obesity (EFO_0001073) from the GWAS catalogue [32]. The genes marked as reported genes from the GWAS catalogue that overlapped with genes in our QTL intervals are listed in Table 5. LEPROT is the candidate gene suggested by the nearest gene, which is also supported by GO and MPD. This gene is associated with human BMI (Table 5). PMAIP1 and FAM155A are DEGs from the RNAseq data set comparing high and low feed efficiency animals. PMAIP1 is associated with human height and BMI. FAM155A is associated with human height and obesity. FAM155A is also the nearest gene in multi-breed meta-analysis. $B M P 2$ is the nearest gene and showed human BMI and height.

\section{Discussion}

Since their emergence, GWAS have improved our understanding of the genetic determinants of complex traits of humans and livestock [33]. In human association studies, the availability of large data sets permits GWAS or metaanalysis on a scale of more than 100,000 individuals [34, 35]. However, in livestock, the scale of the sample size for GWAS is usually smaller than 10,000 from a single source of data $[4,5]$. Here, we performed GWAS with a total sample size of 201,388 animals from three DanBred
Table 5 Human GWAS catalogue for BMI, height, and obesity overlap of genes in QTL intervals for average daily weight gain in three pig breeds and multi-breed meta-analysis

\begin{tabular}{|c|c|c|}
\hline Gene & Human trait & Breed \\
\hline$M C 4 R$ & BMI, Height and Obesity & Yorkshire and Meta \\
\hline PMAIP1 & BMI and Height & Yorkshire and Meta \\
\hline IQCE & Obesity & Yorkshire \\
\hline$A M Z 1$ & Height & Yorkshire \\
\hline GNA12 & Height & Yorkshire \\
\hline$L E P R$ & BMI and Obesity & Landrace \\
\hline LEPROT & $\mathrm{BMI}$ & Landrace \\
\hline SLC17A4 & Obesity & Landrace \\
\hline SLC17A1 & Obesity & Landrace \\
\hline SLC17A2 & Height & Landrace \\
\hline SLC17A3 & Obesity & Landrace \\
\hline TRIM38 & Height & Landrace \\
\hline HIST1H2BD & Height & Landrace \\
\hline BTN1A1 & $\mathrm{BMI}$ & Landrace \\
\hline ZNF322 & Height & Landrace \\
\hline PRSS16 & $\mathrm{BMI}$ & Landrace \\
\hline U6 & Height & Landrace, Yorkshire and Meta \\
\hline$D D \times 42$ & $\mathrm{BMI}$ & Yorkshire and Meta \\
\hline MAP3K3 & Height & Yorkshire and Meta \\
\hline THRB & Height & Yorkshire \\
\hline BMP2 & BMI and Height & Yorkshire \\
\hline DNAJB6 & $\mathrm{BMI}$ & Landrace \\
\hline UBE3C & BMI & Landrace \\
\hline
\end{tabular}

commercial pig breeds. With the increase of the sample size applied for GWAS, we have a higher power to detect the genetic variants that contributes to the trait variation. 
The imputation of the SNP array genotypes to WGS level in humans $[1,36]$ has accelerated the association discoveries and unveiled the underlying genetic determinants of complex traits [16, 37-40]. Recently, similar work has also been accomplished in livestock animals, e.g., dairy cattle $[2,11]$. However, imputation to WGS has been less widely reported in pigs. Most of the work has focused on evaluating the power to impute low-density marker sets $(10 \mathrm{k}, 9 \mathrm{k}$, etc.) to medium-density marker sets (60k) and achieved an imputation accuracy above 0.95 [41-44]. In 2019, van den Berg et al. [45] used two-step strategies (first 80 to $660 \mathrm{k}$, and then to WGS) similar to the strategy followed in the current study. However, the average estimated imputation accuracy for all WGS variants was only around 0.5 for Large White and around 0.4 for Dutch Landrace [45]. In our study, we achieved a much higher estimated imputation accuracy (Fig. 1), which is comparable to that in cattle [2].

In cattle, it is well supported that a multi-breed reference population can achieve higher imputation accuracy [11]. The same strategy has also been applied in pig imputation [45]. Unlike dairy cattle where most animals are purebred, most pigs in the production are two-way crossbred sows or three-way crossbred slaughter animals. Previous work has shown that imputation of a low-density marker set of crossbred to a high-density marker set using a purebred reference population or imputation of a low-density marker set of purebred to a high-density marker set using a crossbred reference population both resulted in high accuracy $[41,43]$. In this study, we tested whether the HD genotypes available from three-way crosses could be used as an intermediate reference panel for imputation from low-density genotyped animals to WGS level to achieve higher accuracy. The results confirmed that the HD crossbred genotypes could be used as an intermediate reference panel for purebreds to impute to WGS variants level.

In this study, we reported the estimated imputation accuracy from Minimac4 instead of calculation of the empirical imputation accuracy. Previous study have shown that the R-sq values estimated by Minimac3 (same as Minimac4) were highly correlated with correlationbased empirical measures [46, 47]. Of course, using the estimated accuracy could limit the direct comparison between studies. However, as imputation becomes a routine work for research groups, scientists are aware of the differences between these two accuracy parameters. Besides this, low imputation accuracy may increase the false negative rate in GWAS, but unlikely to increase false positive rate. Furthermore, the estimated accuracy is sufficient for us to filter out the low quality imputed markers. Therefore, we decided to report the estimated imputation accuracy.
RNA-seq is a powerful tool to carry out functional studies. However, in livestock studies, scientists face the difficulty to choose the right tissue and right development stage related to traits. Previous reports in human found that the estimated correlation of genetic effects of cis-eQTLs between blood tissue and brain tissue could be high as 0.70 [48]. Of course, in such human studies, we could lose some tissue-specific expression genes. Meanwhile, without using the same samples for a GWAS study and a RNA-seq study, it is still worth to combine two types of data [49]. In the above mentioned two human studies, they showed the possibility of using RNA-seq data which are different from the mapping population and with an inconsistency of the study traits and tissues. So, using related-traits RNA-seq data, which are from different breeds could still facilitate finding the common underlying genetic factors between related traits. Therefore, in this study, we used three datasets comparing the DEGs between high and low feed efficiency to prioritize our GWAS result. There was a risk that the difference of the segregation of alleles in different breeds, and the difference between feed efficiency and ADG traits could results in no additional information on candidate genes. Only one of the three RNA-seq datasets showed common genes with our GWAS results.

The GWAS results for three breeds are quite different. The majority of the differences comes from the segregation of different alleles in three breeds, which could be part of the consequence of selection. In the Danbred system, the breeding goal of Duroc is different from the common breeding goal of Landrace and Yorkshire: The goal for Duroc pigs has more weight on growth, leanness, and feed efficiency, whereas the goal for Landrace and Yorkshire has more weight on maternal traits [50]. So certain loci underlying growth may be fixed in Duroc and still segregating in Yorkshire and Landrace. This could be part of the reason why we observed different mapping result between the three breeds. Meanwhile, we also observed differences of the GWAS results between subpopulations. There are two major reason for this: 1 ) these three breeds are under intensive selection; 2) the splitting of the population could reduce the mapping power.

Daily weight gain is a key trait in pig breeding goals since it plays an important role in economic return. The QTL mapped for daily weight gain in pigs was spread across all chromosomes [51]. With the large sample size, we had a high power to locate the QTL. We checked the overlap of the genes in the QTL intervals for daily weight gain pigs with an association reported for three human growth-related traits (human height, obesity, and BMI) from the GWAS catalogue [32]. The underlying logic for this strategy was threefold: 1) the similarity of genetic determinants in pigs and humans; pigs are used as model 
species for human biomedical research [52]; 2) the share of the causal genes may generate new knowledge about gene function [7]; 3) the nearest genes of the lead SNP from GWAS may not be the causal ones. Furthermore, the choice of the human traits was based on two criteria, 1) traits are growth related;2) traits should capture the common causal genes, and so we included child growth trait for these three human traits. Finally, we found at least one gene for each QTL interval for ADG in pigs overlapping with the reported genes for human growthrelated traits.

Human height, BMI and obesity are classical complex traits in human genetics. The accumulation of the knowledge about these trait makes them as a gold mine to understand mammalian growth related traits. By comparing our result with these three traits, we found some genes that are the nearest genes from the GWAS and one gene in the DEGs list. LEPROT is the nearest to the lead SNP on chromosome 6 in Landrace. The GO and MPD have an entry to support that this gene is related to mammalian growth. In a human study, GWAS of 7,215 children revealed that $L E P R O T$ is one of the important loci for early growth [53]. FAM155A is the nearest gene from GWAS of Yorkshire which is also supported by DEGs. This gene was reported in a study for human height, BMI and obesity [54-57]. PMAIP1 is located in the QTL interval, showed in the DEGs list and associated with human BMI and height $[58,59] . B M P 2$ is the nearest gene and literature showed association with human height and BMI [60, 61]. Besides, MRAP2 is an important candidate for ADG in pigs since it has support for GO annotation and Mammalian Phenotype database. ENSSSCG00000036234, ELFN1, LIG4 could also be good candidate genes with support from nearest gene and GO annotation.

\section{Conclusions}

In this study, for three large pig populations, we have accurately imputed from low-density chip to WGS with a high estimated imputation accuracy. This is useful for deciding on an imputation strategy in future genomics studies in pigs. The validation of the QTL interval with the GWAS catalogue of human height, obesity, and BMI showed that GWAS accurately map the QTL region and suggested several candidate genes for daily weight gain in pigs. Our results will improve our understanding of genetic architecture of ADG in pigs and can also be exploited in pig breeding to improve daily weight gain.

\section{Methods}

\section{Animals and phenotype}

Phenotypic records and SNP array genotypes from three DanBred pig breeds, Duroc, Landrace, and Yorkshire, were provided by SEGES - Breeding \& Genetics in pigs. Corrected phenotypes for ADG from $30-100 \mathrm{~kg}$ were computed using predicted effects from the routine genetic evaluation model. The corrected phenotype of $\mathrm{ADG}$ for an individual equals the sum of the predicted breeding value and predicted residual, or in other words the phenotype minus the sum of all predicted nongenetic fixed and random effects.

Briefly, the routine genetic evaluation model is a fourvariate model with traits: average daily gain $7-30 \mathrm{~kg}$, average daily gain $30-100 \mathrm{~kg}$, meat percentage computed from the scanning of back fat and weight at the time of scanning, and feed efficiency. Fixed effects are year-herdmonth (all traits), sex (except for feed efficiency, since only boars have that measurement), and start weight (except for meat percentage). Random effects are breeding value (all traits), pen (ADG, meat percentage), and litter (all traits).

\section{Genotyping and whole-genome resequencing}

In this study, we used three sets of genotypes, starting from low- to medium-density SNP array through to the whole-genome variant level.

\section{Low- to medium-density SNP genotyping}

In total, 201,388 pigs were genotyped with multiple lowto medium-density (8.5 to $70 \mathrm{k}$ ) SNP chips. These were 42,790 Duroc, 88,984 Landrace, and 69,606 Yorkshire. The number of Duroc, Landrace, and Yorkshire animals genotyped with a Genomic Profiler (GGP) Porcine LD array (8.5k) chip was $7,328,13,238$, and 13,282 , respectively; and the number of pigs genotyped with a GGP HD_Porcine chip (43k) was $31,287,68,800$, and 49,313, respectively. The number of pigs genotyped with an Illumina PorcineSNP60 BeadChip (60k) or GGP Porcine HD array (70k) was 4,175 Duroc, 6,946 Landrace, and 7,011 Yorkshire.

\section{High-density (HD) SNP array}

We used high-density genotypes using Affymetrix Axiom PigHD SNP chips (Axiom_PigHDv1, 658k) of 474 threeway crossbred pigs as the intermediate reference panel. The animals were part of the "MetaPig - Modulation of the pig gut metagenome to increase feed efficiency" project (http://www.metapig.eu). These three-way crossbreds are produced by crossing F1 sows from Landrace and Yorkshire inseminated with mixed semen from Duroc boars. The details on this HD genotype data set are presented by Cai et al. [50].

\section{Whole-genome resequencing}

A total of 217 animals of three DanBred commercial pig breeds, i.e., 89 Duroc, 61 Landrace, and 67 Yorkshire, 
were sequenced. The animals for sequencing were selected based on their genetic contribution to the genotyped animals born in 2010, 2011, and 2012. The detail of the sequencing and processing of the data can be found in our previous study [50]. For each individual, pairedend read trimming was performed using trim-fastq from the PoPoolation package [62]. Filtered reads were aligned to the porcine reference genome build 11 [63] by the Burrows-Wheeler Aligner (BWA version 0.7.17) [64], employing "bwa-mem". SAMtools version 1.8 ( $\mathrm{Li}$ et al. 2009) was used for sorting, merging, and marking potential PCR duplicates. From here until the VCF file, the reads were processed using the Genome Analysis Toolkit (GATK version 3.8) [65] according to the 1000 bull genome project pipeline [2].

We applied hard filtering as following. For SNPs, we applied: "QD < 2.0, "SOR > 3.0", "FS > 60.0", "MQ < 40.0", "MQRankSum < -12.5", "ReadPosRankSum < -8.0", and "DP < 4 || DP > 6600". For INDELs, we applied "QD < 2.0", "FS > 200.0", "ReadPosRankSum < -20.0", "InbreedingCoeff $<-0.8$,"DP < 4 || DP > 6600", and "SOR > 10.0". Then we combined the filtered SNP set and INDEL set as the final reference panel.

\section{SNP map position}

The probe sequence (50 bp flanking the SNP) of the $10 \mathrm{k}$, $50 \mathrm{k}, 60 \mathrm{k}$, and HD chip array was mapped to the sus 11.1 assembly by bwa-mem [64]. Only the probes mapped uniquely and CIGAR string with "50M" were retained for the following imputation. We replaced the location of SNPs with the location of the mapping result.

\section{Genotype imputation}

The genotypes (10, 43, 60 and $70 \mathrm{k}$ ) of pigs from all three breeds were combined and phased using Eagle [66]. The HD and WGS marker sets were phased following the procedure of Mesbah-Uddin et al. [67]. Briefly, the genotypes were phased by Beagle4 (r1274) [68] to calculate the genotype probability, and then SHAPEIT2 (v2.r837) [69] was used to call the genotype of the markers with a genotype probability less than 0.99 . For imputation of the combined data set to the WGS level, we adopted two-step imputation strategies described in van Binsbergen et al. and Brøndum et al. [10, 11]. In the first step, we imputed all individuals genotyped by low- and medium-density chips to HD level using the 474 threeway crossbred animals as an intermediate reference panel using Minimac4 [70]. In the second step, we imputed this imputed HD marker set to whole-genome sequencing (WGS) level using the 217 WGS individuals using Minimac4 [70]. Before GWAS, we filtered away all SNPs with minor allele frequency below $0.5 \%$, with a large deviation from Hardy-Weinberg proportions $\left(p<1.0^{-6}\right)$, or an $R^{2}$ value of the estimated imputation accuracy estimated by Minimac4 of less than 0.4 .

\section{Association analysis and meta-analysis}

Due to the large number of animals in each breed, the GWAS by GCTA required a huge amount of computer memory. To deal with this issue, we separated the population into three similar-size subsets based on the birth year (Table 6). Then we run sub-population GWAS in each subset followed by within-breed meta-analysis to combine results from subsets for a breed. For subpopulation GWAS, we estimated the genomic relation matrix (GRM) for all autosomes by GCTA [71] using the imputed HD marker set. The method that was used to estimate GRM between individuals using SNP data is implemented in GCTA [71]. Briefly, genotype dosages and allele frequency of each SNP between one pair of individuals $i$ and $\mathrm{j}$ were used to calculate the relationship score, then average relationship score across all SNPs was calculated as the relationship between individuals $i$ and $\mathrm{j}$. We ran the association study for each chromosome with the GRM obtained above using GCTA [71] in each subset, using a mixed-model approach, GCTA-MLMA using the following model:

$$
y=a+b x+g+e
$$

where $y$ is the phenotype value, $a$ is the population mean, $b$ is the fixed effect the candidate SNP to be tested for association, $x$ is the SNP genotype, and g is the polygenic effect captured by the GRM calculated using the

Table 6 The number of animals, genomic inflation factor (lambda), and the ratio between additive variance (VA) and phenotypic variance (VP), i.e., genomic heritability for each subset of data for three pig breeds

\begin{tabular}{|c|c|c|c|c|c|c|c|c|c|}
\hline & \multicolumn{3}{|l|}{ Duroc } & \multicolumn{3}{|l|}{ Landrace } & \multicolumn{3}{|l|}{ Yorkshire } \\
\hline & Before 2015 & 2015-16 & 2017-18 & Before 2015 & 2015-16 & 2017-18 & Before 2015 & 2015-16 & 2017-18 \\
\hline Number & 15,190 & 12,810 & 14,663 & 22,121 & 18,960 & 46,593 & 22,179 & 16,225 & 29,840 \\
\hline Lambda & 0.95 & 0.95 & 1.00 & 0.98 & 0.93 & 0.97 & 0.92 & 0.92 & 0.92 \\
\hline Lambda & 1.32 & & & 1.30 & & & 1.35 & & \\
\hline$V_{A} / V_{P}$ & 0.252 & 0.149 & 0.175 & 0.282 & 0.299 & 0.228 & 0.282 & 0.250 & 0.230 \\
\hline
\end{tabular}


imputed HD marker set and $e$ is the residual. We set the genome-wide significant threshold as $-\log _{10}$ ( $p$-value) > 8.5 with Bonferroni correction $(0.05 / 13,000,000)$. Then we performed within-breed meta-analysis using METAL [72] with the option of genomic control to deal with the inflation. The number of animals with both genotype and phenotype information was 198,623 (42,663 Duroc, 87,674 Landrace, and 68,244 Yorkshire). At last, we performed multi-breed meta-analysis to investigate the association signals across the three breeds with the same parameter as within-breed meta-analysis. The details of the animal number of each breed, the animal number of each subpopulation, and the genomic inflation factor (lambda) estimated by METAL [72] are listed in Table 6.

\section{Post-GWAS analysis}

The location of the annotated genomic feature was extracted from Ensembl [73]. The GO and KEGG pathway annotation of pig genes were also extracted from Ensembl [73]. We performed the variants annotation using the Ensembl Variant Effect Predictor (ver99) [74]. The possible consequence of the impact of the variant on the phenotype based on mouse mutation lines was retrieved from the Mammalian Phenotype database (MPD) [19].

\section{Differentially expressed gene analysis}

We downloaded three RNA-seq dataset to perform the differentially expressed gene (DEG) analysis: PRJEB23668, PRJEB29969 and PRJEB23289. PRJEB23668 included liver tissue from 20 Maxgro (Hermitage Genetics) x (German Landrace x Large White) pigs. PRJEB29969 included 96 samples from (Large White $x$ Landrace) $x$ Meatline liver or longissimus dorsi muscle tissue. PRJEB23289 included longissimus thoracis muscle tissue from 20 Maxgro (Hermitage Genetics) x (German Landrace $x$ Large White) pigs. Each of the three data sets were divided into high feed efficiency and low feed efficiency groups.

To use the new assembly and annotation information on pigs, we decided to reanalyze three previously reported RNA-seq data from the liver and/or muscle tissue between high and low feed efficiency animals [2931]. The raw reads were downloaded from ENA (https:// www.ebi.ac.uk/eva). We used Trimmomatic (Ver 0.39) [75] to remove potential adapter sequence and trim lowquality reads. For DEG analysis, we downloaded the pig reference genome and transcriptome from Ensembl (v99) [73]. We built a decoy-aware transcriptome index file with the genome sequence and transcriptome sequence following the guidance of Salmon (v1.2.0) [76]. The final clean data were mapped to the decoy transcriptome using Salmon (v1.2.0) [76]. The DEG analysis was performed using DESeq2 [77]. The genes with an adjusted $p$ value $<0.05$ and $\log _{2}$ fold change $>1$ or $<-1$ were regarded as DEGs.

\section{Validation with GWAS catalogue of human height, obesity, and body mass index}

The GWAS catalogue of human height (EFO_0004339withChildTraits_2020_06_10), obesity (EFO_0001073withChildTraits_2020_06_10), and body mass index (BMI, EFO_0004340-withChildTraits_2020_06_04) was downloaded from the NHGRI-EBI Catalog of human genome-wide association studies [32]. The selection of the human traits was limited to growth related. We would like to find the common genetic factors that underlies human height, obesity, BMI and pig ADG on individuals in their growing period. We checked the overlap of the genes in the QTL intervals from our analysis in pigs with the reported genes from the GWAS catalogue in humans.

\section{Abbreviations}

QTL: Quantitative trait loci; GWAS: Genome-wide association study; ADG: Average daily gain; WGS: Whole genome sequencing; HD: High density; MAF: Minor allele frequency; SNP: Single nucleotide polymorphism; GO: Gene ontology; KEGG: Kyoto Encyclopedia of Genes and Genomes.

\section{Supplementary Information}

The online version contains supplementary material available at https://doi. org/10.1186/s12864-022-08373-3.

Additional file 1: Supplementary Figure S1-6.

Additional file 2: Supplementary Table S1.

Additional file 3: Supplementary Table S2.

\section{Acknowledgments}

We acknowledge SEGES Danish Pig Research Centre, Copenhagen, Denmark for giving access to genotype and phenotype data of three pig breeds. MetaPig-Modulation of the pig gut metagenome to increase feed efficiency project is acknowledged for sharing PigHD genotype data for imputation. This research was supported in part by the Center for Genomic Selection in Animals and Plants (GenSAP) funded by Innovation Fund Denmark, grant number 0603-00519B.

\section{Authors' contributions}

GS, ZC, OFC, and MSL conceived and designed the study. ZC, OFC and GS analyzed the data and wrote the paper. MSL, TO and OFC contributed materials and analysis tools. All authors read, revised, and approved the final manuscript.

\section{Funding}

This work is funded by the Center for Genomic Selection in Animals and Plants (GenSAP) funded by Innovation Fund Denmark (grant 0603-00519B). The funders had no input into study design, data analyses and data interpretation.

Availability of data and materials

Genome assembly data used in this study were obtained from the NCBI (https://ftp.ncbi.nlm.nih.gov/genomes/all/GCF/000/003/025/GCF_00000 3025.6_Sscrofa11.1/). All annotation information was obtained from a publicly available source (http://www.ensembl.org). Whole-genome sequences and individual SNP genotype data in this study are available only upon agreement with the breeding organization and should be requested directly from the authors. RNA-seq data were from bio project PRJEB23289, PRJEB23668, and 
PRJEB29969. The genotype and phenotype used and/or analysed during the current study available are available from the authors with the permission of SEGES Danish Pig Research Centre (https://pigresearchcentre.dk). Access to these data for research requires permission from DataGene under a Data Use Agreement.

\section{Declarations}

\section{Ethics approval and consent to participate}

Not applicable because no biological samples were collected and no animal handling was performed for this study. Before this study was conducted, consent from data owners was obtained where required.

\section{Consent for publication}

Not applicable.

\section{Competing interests}

The authors have declared no competing interests.

\section{Author details}

'Center for Quantitative Genetics and Genomics, Aarhus University, 8830 Tjele, Denmark. ${ }^{2}$ SEGES Danish Pig Research Centre, Agro Food Park 15, 8200 Aarhus N, Denmark.

\section{Received: 31 August 2021 Accepted: 8 February 2022}

Published online: 15 February 2022

\section{References}

1. Sudmant PH, Rausch T, Gardner EJ, Handsaker RE, Abyzov A, Huddleston J, et al. An integrated map of structural variation in 2,504 human genomes. Nature. 2015;526(7571):75-81.

2. Daetwyler HD, Capitan A, Pausch H, Stothard P, van Binsbergen R, Brondum RF, et al. Whole-genome sequencing of 234 bulls facilitates mapping of monogenic and complex traits in cattle. Nat Genet. 2014;46(8):858-65.

3. Bouwman AC, Daetwyler HD, Chamberlain AJ, Ponce CH, Sargolzaei M, Schenkel FS, et al. Meta-analysis of genome-wide association studies for cattle stature identifies common genes that regulate body size in mammals. Nat Genet. 2018;50(3):362-7.

4. Cai Z, Guldbrandtsen B, Lund MS, Sahana G. Prioritizing candidate genes post-GWAS using multiple sources of data for mastitis resistance in dairy cattle. BMC Genomics. 2018;19(1):656.

5. Cai Z, Guldbrandtsen B, Lund MS, Sahana G. Prioritizing candidate genes for fertility in dairy cows using gene-based analysis, functional annotation and differential gene expression. BMC Genomics. 2019:20(1):255

6. Cai Z, Guldbrandtsen B, Lund MS, Sahana G. Dissecting closely linked association signals in combination with the mammalian phenotype database can identify candidate genes in dairy cattle. BMC Genet 2019;20(1):15

7. Cai Z, Dusza M, Guldbrandtsen B, Lund MS, Sahana G. Distinguishing pleiotropy from linked QTL between milk production traits and mastitis resistance in Nordic Holstein cattle. Genet Sel Evol. 2020;52(1):19.

8. Cai Z, Guldbrandtsen B, Lund MS, Sahana G. Weighting sequence variants based on their annotation increases the power of genome-wide association studies in dairy cattle. Genet Sel Evol. 2019;51(1):20.

9. Pausch H, Emmerling R, Gredler-Grandl B, Fries R, Daetwyler HD, Goddard ME. Meta-analysis of sequence-based association studies across three cattle breeds reveals 25 QTL for fat and protein percentages in milk at nucleotide resolution. BMC Genomics. 2017;18(1):853.

10. van Binsbergen R, Bink MC, Calus MP, van Eeuwijk FA, Hayes BJ, Hulsegge I, et al. Accuracy of imputation to whole-genome sequence data in Holstein Friesian cattle. Genet Sel Evol. 2014;46(1):41.

11. Brøndum RF, Guldbrandtsen B, Sahana G, Lund MS, Su G. Strategies for imputation to whole genome sequence using a single or multi-breed reference population in cattle. BMC Genomics. 2014;15(1):728.
12. Quan J, Ding R, Wang X, Yang M, Yang Y, Zheng E, et al. Genome-wide association study reveals genetic loci and candidate genes for average daily gain in Duroc pigs. Asian Australas J Anim Sci. 2018;31(4):480-8.

13. Sanchez MP, Tribout T, lannuccelli N, Bouffaud M, Servin B, Tenghe A, et al. A genome-wide association study of production traits in a commercial population of Large White pigs: evidence of haplotypes affecting meat quality. Genet Sel Evol. 2014;46(1):12.

14. Hu ZL, Dracheva S, Jang W, Maglott D, Bastiaansen J, Rothschild MF, et al. A QTL resource and comparison tool for pigs: PigQTLDB. Mamm Genome. 2005;16(10):792-800.

15. Falker-Gieske C, Blaj I, Preuss S, Bennewitz J, Thaller G, Tetens J. GWAS for meat and carcass traits using imputed sequence level genotypes in pooled F2-designs in Pigs. G3-Genes Genomes Genetics. 2019;9(9):2823-34

16. Marouli E, Graff M, Medina-Gomez C, Lo KS, Wood AR, Kjaer TR, et al. Rare and low-frequency coding variants alter human adult height. Nature. 2017;542(7640):186-90.

17. Goumidi L, Cottel D, Dallongeville J, Amouyel P, Meirhaeghe A. Effects of established BMI-associated loci on obesity-related traits in a French representative population sample. BMC Genet. 2014;15(1):62.

18. Brondum RF, Su G, Janss L, Sahana G, Guldbrandtsen B, Boichard D, et al. Quantitative trait loci markers derived from whole genome sequence data increases the reliability of genomic prediction. J Dairy Sci. 2015;98(6):4107-16.

19. Blake JA, Bult CJ, Kadin JA, Richardson JE, Eppig JT, Mouse Genome Database G. The Mouse Genome Database (MGD): premier model organism resource for mammalian genomics and genetics. Nucleic Acids Res. 2011;39(Database issue):D842-8.

20. Sage RD, Atchley WR, Capanna E. House mice as models in systematic biology. Syst Biol. 1993;42(4):523-61.

21. Gorbunova V, Bozzella MJ, Seluanov A. Rodents for comparative aging studies: from mice to beavers. Age (Dordr). 2008;30(2-3):111-9.

22. Peng X, Alfoldi J, Gori K, Eisfeld AJ, Tyler SR, Tisoncik-Go J, et al. The draft genome sequence of the ferret (Mustela putorius furo) facilitates study of human respiratory disease. Nat Biotechnol. 2014;32(12):1250-5.

23. Meeusen EN, Snibson KJ, Hirst SJ, Bischof RJ. Sheep as a model species for the study and treatment of human asthma and other respiratory diseases. Drug Discov Today Dis Model. 2009;6(4):101-6.

24. Gieling ET, Schuurman T, Nordquist RE, van der Staay FJ. The pig as a model animal for studying cognition and neurobehavioral disorders. In: Molecular and functional models in neuropsychiatry. Berlin: Springer; 2011. p. 359-83.

25. Sauleau P, Lapouble E, Val-Laillet $\mathrm{D}$, Malbert $\mathrm{CH}$. The pig model in brain imaging and neurosurgery. Animal. 2009;3(8):1138-51.

26. Meurens F, Summerfield A, Nauwynck H, Saif L, Gerdts V. The pig: a model for human infectious diseases. Trends Microbiol. 2012;20(1):50-7.

27. Diamond LE, Quinn CM, Martin MJ, Lawson J, Platt JL, Logan JS. A human CD46 transgenic pig model system for the study of discordant xenotransplantation. Transplantation. 2001;71(1):132-42.

28. Kanehisa M, Goto S. KEGG: kyoto encyclopedia of genes and genomes. Nucleic Acids Res. 2000;28(1):27-30.

29. Horodyska J, Wimmers K, Reyer H, Trakooljul N, Mullen AM, Lawlor PG, et al. RNA-seq of muscle from pigs divergent in feed efficiency and product quality identifies differences in immune response, growth, and macronutrient and connective tissue metabolism. BMC Genomics. 2018;19(1):791.

30. Horodyska J, Hamill RM, Reyer H, Trakooljul N, Lawlor PG, McCormack UM, et al. RNA-seq of liver from pigs divergent in feed efficiency highlights shifts in macronutrient metabolism, hepatic growth and immune response. Front Genet. 2019;10(117):117.

31. Vigors S, O'Doherty JV, Bryan K, Sweeney T. A comparative analysis of the transcriptome profiles of liver and muscle tissue in pigs divergent for feed efficiency. BMC Genomics. 2019;20(1):461.

32. MacArthur J, Bowler E, Cerezo M, Gil L, Hall P, Hastings E, et al. The new NHGRI-EBI catalog of published genome-wide association studies (GWAS Catalog). Nucleic Acids Res. 2017;45(D1):D896-901.

33. Visscher PM, Wray NR, Zhang Q, Sklar P, McCarthy MI, Brown MA, et al. 10 years of GWAS discovery: biology, function, and translation. Am J Hum Genet. 2017;101(1):5-22.

34. Yang J, Ferreira T, Morris AP, Medland SE, Genetic Investigation of ATC, Replication DIG, et al. Conditional and joint multiple-SNP analysis of 
GWAS summary statistics identifies additional variants influencing complex traits. Nat Genet. 2012;44(4):369-75 S361-363.

35. Rietveld CA, Medland SE, Derringer J, Yang J, Esko T, Martin NW, et al. GWAS of 126,559 individuals identifies genetic variants associated with educational attainment. Science. 2013;340(6139):1467-71.

36. Consortium GP. An integrated map of genetic variation from 1,092 human genomes. Nature. 2012:491(7422):56

37. Yang J, Benyamin B, McEvoy BP, Gordon S, Henders AK, Nyholt DR, et al. Common SNPs explain a large proportion of the heritability for human height. Nat Genet. 2010;42(7):565-9.

38. Zeng J, de Vlaming R, Wu Y, Robinson MR, Lloyd-Jones LR, Yengo L, et al. Signatures of negative selection in the genetic architecture of human complex traits. Nat Genet. 2018:50(5):746-53.

39. Yang J, Bakshi A, Zhu Z, Hemani G, Vinkhuyzen AA, Lee SH, et al. Genetic variance estimation with imputed variants finds negligible missing heritability for human height and body mass index. Nat Genet. 2015;47(10):1114-20.

40. Sniekers S, Stringer S, Watanabe K, Jansen PR, Coleman JRI, Krapohl E, et al. Genome-wide association meta-analysis of 78,308 individuals identifies new loci and genes influencing human intelligence. Nat Genet. 2017:49(7):1107-12.

41. Xiang T, Ma P, Ostersen T, Legarra A, Christensen OF. Imputation of genotypes in Danish purebred and two-way crossbred pigs using low-density panels. Genet Sel Evol. 2015:47(1):54.

42. Badke YM, Bates RO, Ernst CW, Fix J, Steibel JP. Accuracy of estimation of genomic breeding values in pigs using low-density genotypes and imputation. G3. 2014;4(4):623-31.

43. Gualdron Duarte JL, Bates RO, Ernst CW, Raney NE, Cantet RJ, Steibel JP. Genotype imputation accuracy in a F2 pig population using high density and low density SNP panels. BMC Genet. 2013;14(1):38.

44. Cleveland MA, Hickey JM. Practical implementation of cost-effective genomic selection in commercial pig breeding using imputation. J Anim Sci. 2013:91(8):3583-92.

45. van den Berg S, Vandenplas J, van Eeuwijk FA, Bouwman AC, Lopes MS, Veerkamp RF. Imputation to whole-genome sequence using multiple pig populations and its use in genome-wide association studies. Genet Sel Evol. 2019;51(1):2.

46. Hermisdorff IDC, Costa RB, de Albuquerque LG, Pausch H, Kadri NK. Investigating the accuracy of imputing autosomal variants in Nellore cattle using the ARS-UCD1.2 assembly of the bovine genome. BMC Genomics. 2020;21(1):772.

47. Bolormaa S, Chamberlain AJ, Khansefid M, Stothard P, Swan AA, Mason $B$, et al. Accuracy of imputation to whole-genome sequence in sheep. Genet Sel Evol. 2019;51(1):1.

48. Qi T, Wu Y, Zeng J, Zhang F, Xue A, Jiang L, et al. Identifying gene targets for brain-related traits using transcriptomic and methylomic data from blood. Nat Commun. 2018;9(1):2282

49. Zhu Z, Zhang F, Hu H, Bakshi A, Robinson MR, Powell JE, et al. Integration of summary data from GWAS and eQTL studies predicts complex trait gene targets. Nat Genet. 2016;48(5):481-7.

50. Cai Z, Sarup P, Ostersen T, Nielsen B, Fredholm M, Karlskov-Mortensen P, et al. Genomic diversity revealed by whole-genome sequencing in three Danish commercial pig breeds. J Anim Sci. 2020;98(7):skaa229.

51. Hu ZL, Park CA, Wu XL, Reecy JM. Animal QTLdb: an improved database tool for livestock animal QTL/association data dissemination in the postgenome era. Nucleic Acids Res. 2013;41(Database issue):D871-9.

52. Merrifield CA, Lewis M, Claus SP, Beckonert OP, Dumas ME, Duncker S, et al. A metabolic system-wide characterisation of the pig: a model for human physiology. Mol BioSyst. 2011;7(9):2577-88.

53. Couto Alves A, De Silva NMG, Karhunen V, Sovio U, Das S, Taal HR, et al. GWAS on longitudinal growth traits reveals different genetic factors influencing infant, child, and adult BMI. Sci Adv. 2019;5(9):eaaw3095.

54. Kichaev G, Bhatia G, Loh P-R, Gazal S, Burch K, Freund MK, et al. Leveraging polygenic functional enrichment to improve GWAS power. Am J Hum Genet. 2019;104(1):65-75.

55. Zhu Z, Guo Y, Shi H, Liu C-L, Panganiban RA, Chung W, et al. Shared genetic and experimental links between obesity-related traits and asthma subtypes in UK Biobank. J Allergy Clin Immunol. 2020;145(2):537-49.
56. Tachmazidou I, Suveges D, Min JL, Ritchie GRS, Steinberg J, Walter K, et al. Whole-genome sequencing coupled to imputation discovers genetic signals for anthropometric traits. Am J Hum Genet. 2017;100(6):865-84.

57. Wilson CL, Liu W, Yang JJ, Kang G, Ojha RP, Neale GA, et al. Genetic and clinical factors associated with obesity among adult survivors of childhood cancer: a report from the St. Jude Lifetime Cohort. Cancer. 2015;121(13):2262-70.

58. Winkler TW, Justice AE, Graff M, Barata L, Feitosa MF, Chu S, et al. The influence of age and sex on genetic associations with adult body size and shape: a large-scale genome-wide interaction study. PLoS Genet. 2015;11(10):e1005378.

59. Akiyama M, Ishigaki K, Sakaue S, Momozawa Y, Horikoshi M, Hirata M, et al. Characterizing rare and low-frequency height-associated variants in the Japanese population. Nat Commun. 2019;10(1):4393.

60. Kichaev G, Bhatia G, Loh PR, Gazal S, Burch K, Freund MK, et al. Leveraging polygenic functional enrichment to improve GWAS power. Am J Hum Genet. 2019;104(1):65-75.

61. Locke AE, Kahali B, Berndt SI, Justice AE, Pers TH, Day FR, et al. Genetic studies of body mass index yield new insights for obesity biology. Nature. 2015;518(7538):197-206.

62. Kofler R, Orozco-terWengel P, De Maio N, Pandey RV, Nolte V, Futschik A, et al. PoPoolation: a toolbox for population genetic analysis of next generation sequencing data from pooled individuals. PLoS One. 2011;6(1):e15925.

63. Warr A, Affara N, Aken B, Beiki H, Bickhart DM, Billis K, et al. An improved pig reference genome sequence to enable pig genetics and genomics research. bioRxiv. 2019:668921.

64. Ma KC, Mortimer TD, Duckett MA, Hicks AL, Wheeler NE, Sánchez-Busó $\mathrm{L}$, Grad YH. Aligning sequence reads, clone sequences and assembly contigs with BWA-MEM. arXiv preprint arXiv:13033997 2020.

65. Poplin R, Ruano-Rubio V, DePristo MA, Fennell TJ, Carneiro MO, Van der Auwera GA, et al. Scaling accurate genetic variant discovery to tens of thousands of samples. BioRxiv. 2018;201178.

66. Loh PR, Danecek P, Palamara PF, Fuchsberger C, Reshef YA, Finucane HK, et al. Reference-based phasing using the haplotype reference consortium panel. Nat Genet. 2016:48(11):1443-8.

67. Mesbah-Uddin M, Guldbrandtsen B, Lund MS, Boichard D, Sahana G. Joint imputation of whole-genome sequence variants and large chromosomal deletions in cattle. J Dairy Sci. 2019;102(12):11193-206.

68. Browning SR, Browning BL. Rapid and accurate haplotype phasing and missing-data inference for whole-genome association studies by use of localized haplotype clustering. Am J Hum Genet. 2007;81(5):1084-97.

69. Delaneau O, Marchini J, Zagury JF. A linear complexity phasing method for thousands of genomes. Nat Methods. 2011;9(2):179-81.

70. Fuchsberger C, Abecasis GR, Hinds DA. minimac2: faster genotype imputation. Bioinformatics. 2015;31(5):782-4.

71. Yang J, Lee SH, Goddard ME, Visscher PM. GCTA: a tool for genome-wide complex trait analysis. Am J Hum Genet. 2011;88(1):76-82.

72. Speliotes EK, Willer CJ, Berndt SI, Monda KL, Thorleifsson G, Jackson AU, et al. Association analyses of 249,796 individuals reveal 18 new loci associated with body mass index. Nat Genet. 2010;42(11):937-48.

73. Flicek P, Ahmed I, Amode MR, Barrell D, Beal K, Brent S, et al. Ensembl 2013. Nucleic Acids Res. 2013:41 (Database issue):D48-55.

74. McLaren W, Gil L, Hunt SE, Riat HS, Ritchie GR, Thormann A, et al. The ensembl variant effect predictor. Genome Biol. 2016;17(1):122.

75. Bolger AM, Lohse M, Usadel B. Trimmomatic: a flexible trimmer for illumina sequence data. Bioinformatics. 2014;30(15):2114-20.

76. Patro R, Duggal G, Kingsford C. Salmon: accurate, versatile and ultrafast quantification from RNA-seq data using lightweight-alignment. Biorxiv. 2015;021592

77. Love MI, Huber W, Anders S. Moderated estimation of fold change and dispersion for RNA-seq data with DESeq2. Genome Biol. 2014:15(12):550.

\section{Publisher's Note}

Springer Nature remains neutral with regard to jurisdictional claims in published maps and institutional affiliations. 